\title{
EL CONFLICTO EN UCRANIA: EL PRIMER ENFRENTAMIENTO SERIO DE RUSIA CON OCCIDENTE DURANTE LA ETAPA DE LA POST-GUERRA FRÍA
}

\author{
Pablo Telman Sánchez Ramírez
}

\section{INTRODUCCIÓN}

Ucrania se convirtió en un Estado ingobernable de manera inesperada y repentina. De haber sido la república post-soviética más estable desde el punto de vista político, económico y social durante la década de 1990, se ha convertido en una zona inestable, plagada de conflictos armados y resentimientos nacionalistas alentados en cierta medida y, sobre todo, en sus inicios, desde fuera de sus fronteras. En el momento actual (diciembre de 2014), ya se cuenta con más de 5000 muertos y un millón de desplazados como consecuencia de la guerra.

El objetivo del presente artículo es analizar el escenario, las causas, condiciones y la naturaleza del conflicto político, ideológico y bélico en Ucrania, no solamente desde el punto de vista interno del país, sino también desde una óptica más amplia, regional y global, al tomar en cuenta todas las partes involucradas y comprometidas en el avance del diferendo. En este análisis serán imprescindibles las continuas referencias no solamente a los intereses y posturas de Rusia, sino también de Estados Unidos y Europa en relación con Ucrania, así como la importancia que reviste este país para ambas partes, pero también para la propia relación entre ellos. El conflicto en Ucrania y el equilibrio de intereses y poderes de las principales potencias alrededor del mismo tiene connotaciones no solamente regionales, sino también globales al demos- 
trarse que la contención de Rusia se mantiene como una prioridad, en primer lugar de la política exterior de Estados Unidos y que es apoyada por una parte de los países de la Unión Europea. Este bloque mantiene divergencias importantes entre sus miembros en relación con la estrategia que se debe llevar a cabo en relación con Moscú. Toda esta problemática se abordará desde una perspectiva objetiva y analítico-interpretativa, también utilizando una breve pero necesaria referencia histórica. Se realizará un análisis sucinto de la naturaleza y desenvolvimiento de los vínculos bilaterales ruso-ucranianos a lo largo de varios siglos.

El desenvolvimiento acelerado y radical del reciente diferendo en Ucrania demostró hasta qué punto pueden ser importantes los estímulos e influencias externos que llegaron a convertirse en detonante de una situación de conflicto que hasta ese momento yacía latente, pero controlada. Así mismo, el caso de Ucrania demostró una vez más no solamente los mantenidos desacuerdos ideológicos y geopolíticos existentes entre Rusia y Estados Unidos (Europa incluida), sino también el alto nivel de subjetividad que existe en muchos medios de información internacionales, que, lejos de mostrar la realidad desde una perspectiva integral, se limitan a resaltar aristas específicas del conflicto, todo lo cual conduce a presentar una información sesgada e incompleta del asunto en cuestión. Varias fuentes occidentales han intentado mostrar una realidad no completamente objetiva y realista de la situación imperante en la región este de Ucrania.

Luego de las manifestaciones ocurridas en la Plaza Maidán de Kiev (octubre de 2013), producto de la negativa del entonces presidente Víctor Yanukovich de firmar el Pacto de Asociación con la Unión Europea, se inició un proceso de enfrentamientos dentro de la sociedad ucraniana. La atmósfera política interna se polarizó y tensó como nunca antes y fue instaurado un nuevo gobierno de facto que nunca llegó a tener las credenciales necesarias, ni las facultades pertinentes para avanzar en el proceso de negociación y de pacificación nacional. Por el contrario, los representantes del nuevo gobierno interino encabezado por el primer ministro Oleksander Turchinov radicalizaron las posturas de condena contra el expresidente Yanukovich, así como las acusaciones a Rusia por 
apoyar los procesos secesionistas en el Este del país. Sin embargo, las violaciones a los derechos humanos de los ciudadanos que se opusieron al gobierno interino de Kiev en los territorios del Este por parte del nuevo gobierno fueron acalladas en los medios de información nacionales. La conformación de las tropas del nuevo ejército nacional, conocido también como Guardia Nacional, demostró la parcialidad de las nuevas autoridades de Kiev al favorecer la incorporación en sus filas a jóvenes de abierta tendencia neofascista y miembros del conocido grupo Pravyi Sektor (Sector de Derecha), cuyo propósito no era tanto restablecer la paz y el orden en las regiones en conflicto, sino más bien recuperar las ciudades del Este que se habían convertido en el cuartel general de las fuerzas rebeldes separatistas. Estas fuerzas pro-rusas se autodenominaban "milicias de autodefensa" y reclamaban la separación definitiva o, en algunos casos, una federalización del país. De tal forma, se iniciaba un conflicto interno sin precedentes en la vida moderna de Ucrania.

A partir de octubre del año 2013, Ucrania se convierte en la prioridad de la política exterior de Rusia, no solamente en el marco del llamado cercano extranjero (blizhnii zaruviezh), ${ }^{1}$ sino también dentro de la política exterior global del Estado ruso. La abrupta reacción de Moscú ante los hechos ocurridos en Ucrania no resultó una sorpresa ni una improvisación, pues en los postulados y concepto de política exterior adoptados en los años 1999, 2008 y 2013, así como en las doctrinas de defensa y nuclear ${ }^{2}$ se contemplaban situaciones hipotéticas de inestabilidad y conflicto similares a las registradas en Ucrania y que eran consideradas como una amenaza a la seguridad nacional. Durante todos estos años, se ha mantenido

${ }^{1}$ El término "cercano extranjero" fue dado a conocer durante la década de 1990 para identificar a los países que habían formado parte de la Unión Soviética hasta el año 1991.

${ }^{2} \mathrm{El}$ autor analiza en varias de sus publicaciones estas leyes que han servido de marco conceptual, ideológico y práctico a las posturas del gobierno ruso. Se recomienda revisar sus libros: Razón y poder: Rusia, una potencia en el siglo XXI, México, Miguel Ángel Porrúa / Tecnológico de Monterrey, 2005; y El regreso de la eterna Rusia al orden internacional. ¿¿Confrontación o negociación con Occidente?, México, Tecnológico de Monterrey / Montiel \& Soriano editores, 2010. 
una línea realista, nacionalista, pero también pragmática dentro de la política exterior y de seguridad nacional de Rusia.

Entre los objetivos fundamentales de la actual doctrina de política exterior rusa se encuentran el mantener una estrategia de protección de los intereses nacionales del Estado ruso que no fomente la confrontación con otros Estados, así como la protección de los derechos humanos e intereses legítimos de los ciudadanos rusos y también de los paisanos residentes fuera del país. ${ }^{3}$ Actualmente, se encuentran más de doce millones de ciudadanos de origen ruso en Ucrania, que tanto desde el punto de vista cultural, como lingüístico y étnico se mantienen estrechamente vinculados con el país vecino. Rusia no llegó a invadir a Ucrania y de hecho retiró sus tropas de 50000 efectivos que tenía desplegadas en las fronteras comunes, para facilitar el proceso de elecciones que se llevó a cabo en mayo de 2014, así como el cumplimiento de los acuerdos que recién habían sido firmados en Ginebra.

\section{ANTECEDENTES históricos}

Para entender la dimensión y el alcance de la estrategia desplegada por el gobierno ruso ante el escenario de inestabilidad y conflicto del país vecino, se deben tomar en cuenta los antecedentes históricos de la compleja, pero a la vez estrecha, interdependiente e intensa relación bilateral que ha existido entre Rusia y Ucrania a lo largo de más de mil años, desde la creación de la Kievskaya Russ (Rusia Kievita) en el siglo Ix. El territorio conocido como Ucrania tiene un pasado plagado de guerras y continua división tanto étnica, como territorial, política e ideológica. El pasado de Ucrania puede ser comprendido con base en la historia de varios imperios europeos, incluido el otomano. Dentro de las conquistas del Imperio Otomano se encontraba el Kanato de Crimea, cuyo territorio era ocupado por una mayoría tártara. Este territorio perteneció a

${ }^{3}$ Concepto de la Política Exterior de la Federación Rusa (traducción no oficial del ruso), Boletín informativo del Ministerio de Asuntos Exteriores de la Federación Rusa, Departamento de Información y Prensa, Moscú, 18 de febrero de 2013, p. 6. 
los otomanos hasta el año 1774, posteriormente se efectuó la anexión de Crimea al Imperio Ruso en 1783, durante el gobierno de la emperatriz Catalina la Grande. Por ejemplo, en el caso de Crimea y las acusaciones de anexión por parte de Rusia, habría que señalar que esta península fue la base naval de la fuerza marítima del imperio ruso desde el siglo xviII, cuando Catalina la Grande dispuso la anexión de la península al imperio ruso, luego de la victoria sobre el Imperio Otomano. Con Catalina la Grande se emprendieron varias campañas de "rusificación" mediante las cuales se influyó de manera cultural y política a la población de Ucrania para que adoptaran las costumbres rusas, incorporando a los practicantes de la Iglesia Uniata dentro de la Iglesia Ortodoxa. En el lado oeste de Ucrania ha existido siempre una mayor identificación cultural, histórica e ideológica con una raíz europea occidental debido a la pertenencia de estos territorios a Polonia y al Imperio Austro-Húngaro a partir del siglo xviII. Por el contrario, la parte oriental de Ucrania tiene un contexto mucho más cercano a Rusia a partir de su conquista por parte del Imperio Ruso tras las particiones de Polonia. Durante gran parte del siglo xix, fueron prohibidas las publicaciones en ucraniano, así como la alfabetización en esta lengua en el territorio ucraniano.

Durante los primeros años de la existencia de la Unión Soviética (durante el mandato de Lenin fundamentalmente), se llevó a cabo una política de inclusión de las etnias no rusas dentro del incipiente Estado soviético. Como consecuencia de esta estrategia multicultural del Estado bolchevique, se permitió el uso, estudio y publicación de obras en lengua ucraniana. Por el contrario, a partir de 1924 y durante el régimen de Iosif Stalin se adoptó una política de rusificación forzosa, en la que se persiguió al nacionalismo ucraniano de manera extremista y sanguinaria.

En 1954, Nikita Jruschov tomó la decisión de ceder el territorio estratégico de Crimea a la República Socialista Soviética de Ucrania. La dirigencia soviética jamás imaginó que exactamente sesenta años después de haber adoptado esta decisión se desataría un conflicto de tal magnitud entre Rusia y Ucrania por ese enclave estratégico, que también guarda un valioso legado histórico producto de la victoria soviética sobre el fascismo en el año 1945. Finalmente, 
el hecho de ceder la región de Crimea a Ucrania fue un hecho ilegítimo debido a que el Presidium del Soviet Supremo de la Unión Soviética no tenía las facultades necesarias para ceder territorios de una república soviética a otra y ese acontecimiento contradijo los postulados de la Constitución de la URSS de 1937. Se demostraba una vez más el poder de decisión que tenía la figura del Presidente del Buró Político del pcus, del Presidium del Soviet Supremo y del Comité Central, que como dijimos en ese momento recaía en Nikita Jruschov.

Durante el mandato de Mijaíl Gorbachov se abrieron las oportunidades para que los pueblos y nacionalidades que formaban parte de la Unión Soviética adquirieran un espacio para defender sus intereses y preocupaciones nacionales. Así comenzó el fin del Estado soviético al tomar fuerza las tendencias separatistas, que finalmente vencieron al régimen soviético con la desintegración de la Unión Soviética.

El 20 de enero de 1991 se realizó un referéndum en Crimea en el cual la mayoría aplastante de los votantes (93.26\%) se pronunció a favor de la reconstrucción de la República Socialista Soviética Autónoma de Crimea como sujeto de la URSS y miembro del Tratado de la Unión. Esta consulta popular demostró la inconformidad de los ciudadanos con el traspaso de su territorio a Ucrania en 1954 y con la abolición en 1945 de la República Autónoma Socialista Soviética de Crimea y la creación en su lugar de la región de Crimea. Sin embargo, a finales de ese mismo año desaparecía la URSS como Estado y Ucrania y Rusia se convertían en dos países independientes, por lo que Crimea permanecería bajo el control de Ucrania.

En 1992, la Federación Rusa reclama la devolución de Crimea a lo que el gobierno de Leonid Kravchuk se opone y le conceden el estatuto de república autónoma. En 1997 se firma un acuerdo para que Rusia conservase la base naval de Sebastopol y otras instalaciones militares de Crimea por un plazo de veinte años. ${ }^{4}$ En 2010, con la llegada de Víctor Yanukovich, se firma un acuerdo

${ }^{4}$ Pablo Telman Sánchez Ramírez, El regreso de la eterna Rusia al orden internacional, pp. 134-135. 
con Rusia para extender hasta el año 2042 la permanencia en Crimea de la Flota Rusa del Mar Negro, a cambio de la rebaja en un $30 \%$ del precio del gas para Ucrania.

\section{Posturas oficiales de la Unión Europea y de Estados UNIDOS EN RELACIÓN CON EL CONFLICTO EN UGRANIA}

Es importante señalar que las posturas de apoyo llevadas a cabo por representantes de la Unión Europea y de gobiernos occidentales -incluido Estados Unidos-, en relación con las manifestaciones antigubernamentales en Kiev funcionaron como un detonante mayor, que lejos de lograr un apaciguamiento del descontento popular, sirven para acelerar el proceso de caída del régimen del corrupto y autoritario, pero también electo democráticamente presidente Yanukovich. Como dijimos, la causa principal de las revueltas lo constituyó la negativa de Yanukovich a firmar un acuerdo con la Unión Europea. Al cabo de siete meses de conflicto, se logró finalmente firmar un acuerdo similar con la Unión Europea (27 de junio de 2014) que también incluiría a Georgia y Moldavia. ${ }^{5}$

A pesar de que la política exterior de Ucrania durante todo el mandato del presidente Víctor Yuschenko (2004-2010) estuvo concentrada en mantener como prioridad sus nexos con Europa y Estados Unidos, la respuesta de la contraparte, sobre todo de la Unión Europea, resultó cautelosa y denotó falta de interés hacia la profundización de los vínculos con Ucrania. Una de las razones fundamentales de esta estrategia europea era la falta de credibilidad del gobierno ucraniano a causa de escándalos de corrupción y vacío de poder en que se vio envuelto. Asimismo, la derrota sufrida por el partido del presidente frente a su opositor -a sólo dos

${ }^{5} \mathrm{El}$ acuerdo firmado con Ucrania establece un área de libre comercio bilateral y la desaparición de aranceles entre la Unión Europea y este país. Así mismo, se negocia un acuerdo de eliminación de visados que debe aprobarse en el futuro cercano. Véase Lucía Abellán, "Ucrania y la UE firman el pacto de libre comercio que desató la crisis con Rusia”, El País, 27 de junio de 2014, en http:/ internacional. elpais.com/internacional/2014/06/27/actualidad/1403853122_102740.html [2 de julio de 2014]. 
años de su arribo al poder- en las elecciones parlamentarias de marzo del 2006, demostraron el rápido desencanto de la población ante el régimen de Yuschenko, lo cual provocó aún mayor cautela por parte de Europa. La Unión Europea promovió una negociación con los países post-soviéticos para buscar una solución que respondiera a sus intereses de integración, pero que no afectara sus relaciones con Rusia: la creación de la Asociación Oriental. La Unión Europea ratificó el 7 de mayo del 2009 en Praga la creación de la Asociación Oriental, que, a propuesta de Polonia y de Suecia, pretendió profundizar las relaciones bilaterales del bloque comunitario con sus vecinos postsoviéticos europeos: Ucrania, Moldavia, Bielorrusia, Georgia, Armenia y Azerbaiyán. La propuesta de Asociación Oriental representó una manera inteligente de estrechar los vínculos con estos países, al tiempo que no se les ofrecía la membrecía inmediata ni permanente en la Unión Europea. Esto resultaba provechoso tanto para la Unión Europea como para Rusia, que no veía de buena manera la entrada de estos países en la Unión Europea. Sin embargo, el objetivo de estos países y, en primer lugar, la Ucrania de Yuschenko, era convertirse en miembros permanentes de la organización europea.

La estrategia del gobierno de Yanukovich mucho más cercana a Rusia que a Occidente, así como su decisión de interrumpir y rechazar de manera definitiva el proceso de negociación con la Unión Europea y continuar adelante con el proceso de firma de un acuerdo económico, comercial y energético con Moscú que facilitaría su posterior entrada en la Unión Económica de Eurasia incomodaba a las autoridades comunitarias europeas. Los llamados a respetar el orden democrático, los derechos humanos de los ciudadanos y el sentir popular antigubernamental fueron los mayores reclamos de la Unión Europea a Ucrania. Precisamente las tensiones y roces que prevalecen desde hace varios años en las relaciones bilaterales de países como Gran Bretaña, Polonia, Lituania, Rumania, Suecia, Bulgaria (recién anunció la suspensión de la construcción en su territorio del gasoducto South Stream proveniente de Rusia) con Rusia predeterminaron el apoyo más entusiasta y activo de parte del bloque comunitario hacia las fuerzas de oposición al gobierno pro-ruso de Kiev, así como la imposición de sanciones 
más severas a Rusia desde el inicio, mientras que Alemania -dependiente del gas ruso y con un liderazgo regional favorecido por la posguerra fría- una vez más demostraba una postura conciliadora y mediadora hacia Rusia, sobre todo en los inicios del conflicto, sin dejar de mantener su apoyo formal a la política comunitaria de presión, advertencias diplomáticas e incluso aplicación de sanciones económicas, políticas y militares contra el gobierno de Moscú. Alemania y Francia fueron los dos Estados comunitarios con los que Rusia mantuvo más contactos bilaterales desde el inicio.

\section{SANCiONES ECONÓMicas y COMERciales de Estados Unidos y La Unión Europea contra Moscú}

Varios especialistas dentro y fuera de Rusia consideran que las sanciones contra Moscú han resultado contraproducentes y exageradas. Entre ellos destaca Andrei Illarionov, quien fuera asesor de política económica del gobierno de Putin entre los años 2000-2005. Illarionov pone en duda la eficacia de las sanciones establecidas en contra de Rusia y en la Conferencia de Seguridad Globsec en la capital eslovaca, dijo "que en el Kremlin sólo se ríen al respecto [...] Ellos piensan que es una broma, nadie lo toma en serio. Las sanciones no son efectivas. Son totalmente inadecuadas sobre la base de los objetivos del Kremlin". ${ }^{6}$ No coincido plenamente con este análisis, pues la economía rusa ya se está viendo afectada como resultado de estas sanciones, aunque el gobierno de Putin ha establecido nuevos nexos comerciales con empresas de otras regiones como Asia y América Latina. Así mismo, si de hablar de sanciones se trata y de sus efectos, lo primero que habría que analizar es si el objetivo principal de las mismas sería conservar la integridad territorial de Ucrania y lograr la pacificación de los territorios rebeldes. Si éste fuese el propósito, entonces las sanciones contra Rusia no son una

\footnotetext{
${ }^{6}$ Karl-Peter Schwarz, "Krise in der Ukraine. Im Kreml lacht man über Sanktionen" ("El Kremlin se ríe de las sanciones"), Pressburg, Alemania, 15 de mayo de 2014, en http://www.faz.net/aktuell/politik/europaeische-union/krise-in-derukraine-im-kreml-lacht-man-ueber-sanktionen-12940541.html
} 
vía adecuada; precisamente esto es lo que ha demostrado el desenvolvimiento de los acontecimientos durante los últimos seis meses. Por el contrario, el conflicto se ha recrudecido y las partes enfrentadas mantienen sus posturas invariables, lo que aleja la posibilidad de una negociación exitosa. El académico y politólogo estadounidense John J. Mearsheimer (Universidad de Chicago) considera como "un gran y fatal error la postura del presidente Obama de imponer sanciones severas a Rusia y apoyar al nuevo gobierno en Ucrania”, lo cual en su opinión está basado en la misma lógica errada que tuvo la Casa Blanca desde el inicio del conflicto al apoyar a los manifestantes opositores en Kiev, todo lo cual no hizo más que precipitar la crisis. ${ }^{7} \mathrm{El}$ citado autor, quien es especialista en las políticas de las Grandes Potencias, ${ }^{8}$ considera que "la causa principal de la crisis actual en Ucrania es la posible expansión de la OTAN y el compromiso de Washington de alejar a Ucrania de la esfera de influencia de Rusia e integrarlo a Occidente". ${ }^{9}$ Rusia aceptó de mala gana la incorporación al bloque militar de los países exsocialistas de Europa, incluidos los Estados bálticos; pero cuando se anunció la posible entrada en octubre del 2008 de Georgia, Ucrania y Moldavia, que ya había sido ratificada por el gobierno de George W. Bush, Rusia negoció de manera exitosa con Alemania y Francia para que no apoyaran este proceso de entrada a la OTAN.

El segundo paquete de sanciones económicas y comerciales contra Rusia anunciadas por el gobierno de Obama, y posteriormente por la Unión Europea, se vislumbra más enfocado a afectar de manera directa a sectores claves de la economía rusa, tales como el energético, militar y financiero-comercial. Se ampliaron las sanciones occidentales inicialmente a tres de los bancos más importantes de Rusia: Banco de Moscú, Banco Agrícola y vтв y posteriormente a los demás bancos con participación estatal (Sberbank,

${ }^{7}$ John J. Mearsheimer, "Getting Ukraine Wrong", The International New York Times, 14 de marzo de 2014.

${ }^{8}$ Este académico también escribió el libro titulado La tragedia de la política de las grandes potencias (The Tragedy of Great Power Politics), Nueva York, W. W. Norton, 2001.

${ }^{9}$ Mearsheimer, op. cit. 
y Gazprombank), así como el bloqueo de exportaciones de bienes específicos y tecnologías al sector de defensa y energético nacional. También fueron suspendidos los créditos que fomenten las exportaciones hacia Rusia y la financiación de proyectos de desarrollo económico en Rusia, así como el embargo de armas hacia este país por parte de países miembros de la Unión Europea. Rusia exporta armamento a este bloque por un valor de 3200 millones de euros, mientras que los países comunitarios sólo venden a Moscú 300 millones y Francia es el principal exportador. ${ }^{10}$ Todas estas nuevas sanciones podrían llegar a afectar a la economía rusa en un mediano plazo, sobre todo las provenientes de los países miembros de la Unión Europea, con los que Rusia mantiene nexos económicos, comerciales y financieros mucho más importantes y permanentes que con Estados Unidos.

La Rusia de Putin observa el desenvolvimiento del conflicto en Ucrania desde una óptica puramente geopolítica e ideológica; desde ese espectro de análisis, el alejamiento geopolítico de este país de la influencia de Rusia y su acercamiento a la otAn, a la Unión Europea y, sobre todo, el estrechamiento de relaciones económicas, políticas y militares con Estados Unidos, es considerado una amenaza directa a su seguridad nacional. ¿Qué postura asumiría la Casa Blanca si repentinamente México se convirtiese en un Estado hostil hacia nuestro vecino del norte y renunciase a los convenios ya ratificados previamente, tales como el TLCAN?

Por el contrario, el gobierno de Estados Unidos necesitaría fomentar relaciones de cooperación y confianza con Rusia -lo cual cada vez se ve más remoto e improbable con las presiones políticas, económicas, comerciales y militares directas del presidente Obama hacia Putin-, para actuar de conjunto y de manera coordinada en relación con otros conflictos y escenarios en los cuales el gobierno ruso presenta ventajas geopolíticas, como son los casos de Siria, Irán, Iraq, Afganistán, Asia Central e incluso la propia China. El régimen de Putin se puede definir como un régimen semiautoritario que defiende las tesis del nacionalismo, el patriotismo, la multi-

${ }^{10}$ Lucía Abellán, "La UE propone sanciones a Rusia que superan los 10.000 millones”, El País, España, 24 de julio de 2014. 
polaridad y el pragmatismo, pero no resalta ni el comunismo, ni el marxismo-leninismo como era el caso de los regímenes soviéticos. Por el contrario, el régimen comunista de China sí mantiene vigentes estos principios en sus discursos oficialistas; sin embargo, esto no inquieta a Occidente como para aplicar una política de sanciones similares a las empleadas contra Moscú. China podría llegar a convertirse en el verdadero rival -no solamente económico y comercial- de Estados Unidos, pero con las posturas equivocadas y altaneras del presidente Obama hacia Moscú en relación con el conflicto en Ucrania es muy probable que se logre fomentar una duradera relación de enemistad y fricción también con Rusia, todo lo cual finalmente afectaría al liderazgo estadounidense en el escenario mundial. Dimitri Simes señala que "con la actual postura belicista del presidente Obama en relación con Rusia se ha logrado obtener lo peor de todos los mundos: el fortalecimiento del nacionalismo militante desde dentro de Rusia, convencer a Putin de que Estados Unidos es débil e indeciso, así como exponer y evidenciar las divisiones dentro de Occidente. ${ }^{11}$ Finalmente, estos tres factores debilitan a Estados Unidos y fortalecen a Rusia. ¿Quién sale ganando entonces? Evidentemente el segundo.

Un mayor acercamiento entre Rusia y China será un lógico resultado de la equivocada postura estadounidense. Uno de los mejores ejemplos de las declaraciones y actuaciones abiertamente anti-rusas del presidente Obama lo constituyó precisamente su viaje oficial a Polonia (3 de junio de 2014) en ocasión del 25 aniversario de la derrota del régimen comunista en ese país y su discurso en el que destacaba que "he venido a Varsovia en nombre de Estados Unidos, en nombre de la OTAN, para reafirmar nuestro compromiso inquebrantable con la seguridad de Polonia. El artículo 5 [de la OTAN] es claro: un ataque a uno de nosotros es un ataque a todos". Su tono beligerante y de amenaza velada iba dirigido contra el gobierno ruso, lo cual nos recordó la Guerra Fría.

${ }^{11}$ Dimitri Simes, "How Obama Is Driving Russia and China Together", The National Interest, 24 de junio de 2014, en http:/ / nationalinterest.org/feature/howobama-driving-russia-china-together-10735 


\section{Estrategia de Estados Unidos y la OTAN EN LA REgión}

La estrategia actual del presidente Obama es aumentar los ejercicios de entrenamiento militar y la presencia de soldados estadounidenses y de la oTAN en los países de Europa del Este fronterizos con Rusia, o sea, llevar a cabo una estrategia que es considerada por Rusia como una amenaza directa a su seguridad nacional. En 2014, Estados Unidos mantiene 21 bases militares en Europa, pero lo que más preocupa a Rusia es que desde abril este país tiene estacionados más de 600 soldados en Polonia, Lituania, Letonia y Estonia, o sea, en su propia frontera noroeste. Washington también anunció el aumento de la participación de su Marina en los despliegues navales de la otan en el Mar Mediterráneo y el Mar Negro, para ir en ayuda de "amigos cercanos", como Georgia, Moldavia y Ucrania, países que son considerados por Rusia como su zona de influencia directa. Finalmente, los ministros de Defensa de la OTAN, reunidos en Bruselas (junio de 2014), apoyaron el plan de Obama al considerarlo como "otra señal de liderazgo y determinación” dentro de la отAN. ${ }^{12}$ Todo esto no hace más que reforzar la paranoia del Kremlin y su patológica desconfianza ante Occidente, que por momentos se torna lógica y fundamentada, a la vez que refuerza su discurso nacionalista y patriótico dentro del país para justificar sus posturas militaristas. Ucrania siempre ha sido un objetivo clave para la OTAN por tener el segundo ejército más numeroso de Europa (después del ruso) y contar con la frontera europea más extensa con Rusia, pero luego del fallido intento de alejamiento de Rusia con la Revolución Naranja en el año 2004 no se les había presentado una oportunidad como ésta.

Es evidente que para Occidente -Estados Unidos en primer lugar- es preferible una Ucrania gobernada por fuerzas ultranacionalistas de extrema derecha, que podrían resultar inestables y poco confiables, antes que aceptar un gobierno subordinado a

12 A. Tapia, "Obama busca contener a Rusia y anuncia más tropas para Europa”, La Tercera (Chile), 4 de junio de 2014, en http://diario.latercera.com/2014/ 06/04/01/contenido/mundo/8-166017-9-obama-busca-contener-a-rusia-y-anuncia-mas-tropas-para-europa.shtml 
Rusia, como fue el caso de Yanukovich. Los objetivos geoestratégicos de Occidente son aislar territorial y militarmente a Rusia de manera definitiva, al costo que sea necesario y, en este caso, Ucrania ha sido el escenario propicio. Se mantienen impávidos ante un escenario golpista que colocó en el poder a unos personajes que no representan de manera democrática a todos los sectores de la sociedad ucraniana y que incluso llegaron a pronunciarse por la eliminación del idioma ruso como segunda lengua oficial del Estado, sin tomar en cuenta los derechos e intereses de millones de ciudadanos del país. El gobierno de Obama juega también la carta doméstica dentro de su propio país al intentar convencer a la opinión pública estadounidense, pero también al partido republicano, de su liderazgo internacional y de su mano firme frente a Rusia, luego de haber sido tan criticado por haber claudicado ante el gobierno de Putin en el caso de Siria.

\section{Búsqueda DE Nuevos SOCIOS POR PARTE DE RUSia EN OTROS ESCENARIOS REGIONALES}

Por su parte, el gobierno ruso avanza en la búsqueda de otros potenciales socios estratégicos en escenarios de América Latina y el Caribe, Medio Oriente, Asia Central, Asia Pacífico e Indochina. La rapidez con que ha avanzado la firma de acuerdos con China en diferentes esferas, pero sobre todo en la energética, constituyen el mejor ejemplo. También con Brasil y Argentina se ha agilizado la ratificación de nuevos acuerdos en cuestiones del comercio bilateral. Rusia demuestra también su intención de mirar hacia otros horizontes en Eurasia y con los miembros de los BRICs. De tal forma, empieza a fomentar operaciones comerciales en otras monedas diferentes al dólar, tales como el yuan, euro e incluso el propio rublo (que ya se utiliza como divisa comercial con los países postsoviéticos). En junio, el diario estadounidense The Financial Times señaló que algunas empresas rusas estaban preparándose para reorientar sus contratos internacionales y pagar las operaciones en 
yuanes y otras divisas asiáticas, como el dólar de Hong Kong y el dólar de Singapur. ${ }^{13}$

Gazprom y la empresa china CNPC suscribieron en mayo del 2014 el mayor contrato en la historia de la empresa gasífera rusa para el suministro a China de 38000 millones de metros cúbicos de gas anuales durante los próximos treinta años, por un monto de 400000 millones de dólares. Fueron firmados más de cuarenta documentos conjuntos en las ramas del transporte, energía, infraestructuras, industria automovilística y construcción aeronáutica. China es el principal socio comercial de Rusia en el mundo. La circulación de mercancías entre ambos países alcanza la cifra de 90000 millones de dólares anuales. ${ }^{14}$ Ambos gobiernos aumentarán el porcentaje de pagos recíprocos en rublos y yuanes a partir del presente año.

Luego de la segunda ronda de sanciones de la Unión Europea contra Rusia, es muy probable que este país responda con nuevas contramedidas entre las que ya se contemplan la prohibición de importaciones de autos, ropa, así como la restricción de la participación de empresas extranjeras en los contratos públicos para el suministro de fármacos al mercado ruso. Las contramedidas rusas podrían incluir la expulsión de compañías europeas de importantes proyectos que llevan a cabo en territorio ruso, lo cual beneficiaría a los inversores de otros países. Por ejemplo, la banca china ya tiene planes de financiar las obras de una línea ferroviaria de alta velocidad entre Moscú y Kazán. ${ }^{15}$

Por otra parte, se debe resaltar la postura asumida por la canciller alemana Angela Merkel, quien demostró una estrategia moderada hacia Moscú, sin dejar de apoyar la línea de Occidente de

13 Alexei Druzhinin, "Gazprom busca cotizar en la bolsa de Hong Kong", Agencia de noticias RIA-Novosti, Moscú, 26 de junio de 2014, en http:/ /sp.ria.ru/ economy/20140626/160562654.html

${ }^{14}$ Alexei Druzhinin, "Gazprom firma el contrato de gas con China”, Agencia de noticias RIA-Novosti, Shanghái, 21 de mayo de 2014, en http://sp.ria.ru/economy/20140521/160155188.html

${ }^{15}$ Christian Lutz, "Cómo Rusia responderá a las nuevas sanciones de la Unión Europea”, Agencia de noticias RIA-Novosti, Moscú, 12 de septiembre de 2014, en http://sp.ria.ru/revista_de_prensa/20140912/161793263.html 
presionar al gobierno ruso. En varias de sus entrevistas, Merkel reiteró que las sanciones financieras, económicas y militares contra Moscú no han tenido ningún resultado:

Esto hasta el momento no ha tenido éxito, y no se va a ver en el futuro. Los europeos y sus socios transatlánticos discuten, por supuesto, pero después actuamos juntos. La Comisión Europea está preparando propuestas que tengan en cuenta las diferentes preocupaciones de los distintos Estados con base en su dependencia del gas ruso. Nadie en Europa está a la espera de sanciones, nuestro objetivo siempre ha sido una solución diplomática a la crisis. Las sanciones no son un fin en sí mismo para nosotros. ${ }^{16}$

Sin embargo, esa voz negociadora y cautelosa ha dejado lugar a un régimen de sanciones por parte de la Unión Europea y Estados Unidos hacia Rusia, lo cual a su vez provocó el anuncio de las contrasanciones rusas (agosto de 2014), sobre todo contra productos de exportación que la Unión Europea hace llegar a Rusia, como frutas, verduras, productos cárnicos de todo tipo y lácteos. Por el momento, no se ha anunciado ninguna limitación de Rusia en la exportación de gas y petróleo hacia los países de la Unión Europea. Quizás la carta energética sea un recurso que el gobierno ruso utilizará hasta el último momento, pues continúan avanzando las negociaciones paralelas con China en estas cuestiones.

Sin embargo, Moscú mostró una señal de pragmatismo "a corto plazo", luego de la firma de un acuerdo (30 de octubre de 2014) con Ucrania y la Unión Europea que permitiría reanudar el suministro de gas a Kiev, interrumpido desde hacía cuatro meses, y mantenerlo al menos hasta marzo de 2015, lo cual a su vez colocó una presión sobre el gobierno ucraniano frente a su homólogo ruso. Por su parte, Ucrania promete pagar 3100 millones de dólares por deudas acumuladas con Rusia desde el inicio del conflicto.

${ }^{16}$ Hubert Meinung, "Russland wendet sich wieder altem Denken zu“, ("Rusia regresa al antiguo pensamiento”, en alemán), entrevista realizada para F.A.Z. interview, Alemania, 16 de mayo de 2014. Disponible en internet en idioma alemán: http:/ / www.faz.net/aktuell/politik/kanzlerin-merkel-im-f-a-z-gespraech-russlandwendet-sich-wieder-altem-denken-zu-12941544.html 
Sin embargo, esa misma semana surgió un clima de máxima tensión entre Rusia y Occidente, después de que quince aviones militares rusos penetrasen el cielo europeo y provocasen una reacción de la OTAN, que anunció el "refuerzo" de la vigilancia aérea. ${ }^{17}$

\section{El tema de Crimea y el Reavivar del Sentimiento NAGIONALISTA EN RUSIA}

El gobierno ruso continúa aprovechando la veta nacionalista-patriótica de su política. En el cuestionado aspecto de la anexión de Crimea, el gobierno de Putin recibió el apoyo prácticamente unánime, no sólo por parte de los ciudadanos de su país, sino incluso de los partidos de oposición y de todos los diputados del Consejo de la Federación, que autorizaron de manera unánime al presidente Putin a inicios de marzo el uso de la fuerza militar dentro del territorio de Ucrania, para salvaguardar los derechos y la integridad física de los ciudadanos rusoparlantes en el territorio vecino. ${ }^{18}$ La prominente activista opositora e influyente personalidad de los medios rusos Ksenia Sovchak aprobaba desde un inicio la gestión de Putin en el conflicto en Crimea: "Si Putin logra el retorno de Crimea a Rusia sin derramamiento de sangre, él será recordado como una gran figura histórica dentro de nuestro país y nadie podrá evitarlo". ${ }^{19}$ Por el contrario, en Occidente y, sobre todo en

${ }^{17}$ Ignacio Fariza, "Rusia y Ucrania sellan el acuerdo para cerrar la guerra del gas”, El País, 31 de octubre de 2014, en http://internacional.elpais.com/internacional/2014/10/30/actualidad/1414701076_728776.html

${ }^{18}$ De acuerdo con la Constitución rusa, el uso de la fuerza militar nacional en territorios extranjeros sólo puede ser autorizada por la mayoría de los miembros del Consejo de la Federación sobre la base de una solicitud del presidente. Igor Rozin, "Putin solicita a los senadores la aprobación para el uso de la fuerza militar en Ucrania", Rossiyskaya Gazieta (Moscú), 1 de marzo de 2014, en http://es.rbth. com/noticias/2014/03/01/putin_pide_al_senado_ruso_autorizacion_para_emplear_tropas_rusas_en_37783.html [23 de junio de 2014].

${ }^{19}$ Andrew Osborn, “Crimea, a Symbol of Russian Identity Vital for Putin's Legacy”, 13 de marzo de 2014, cable de Agencia de Noticias Reuters, en http://in.re uters.com/article/2014/03/13/ukraine-crisis-crimea-putin-idINDEEA2C08H2014 0313 
Estados Unidos, los ciudadanos consideran al Estado ruso como una continuación de la Unión Soviética "por su naturaleza agresiva e impredecible". ${ }^{20}$ De acuerdo con una encuesta del periódico The Wall Street Journal y el canal NBC News, 72\% de los estadounidenses consideran a Rusia un adversario y solamente $5 \%$ ven a Putin como un líder confiable y positivo, mientras que $63 \%$ lo consideran una figura negativa. ${ }^{21}$

Los resultados abrumadoramente favorables a la separación de las regiones de Donietsk y Lugansk del territorio de Ucrania como resultado del referendo celebrado el 11 de mayo pasado reforzaron las posturas nacionalistas de la contraparte rusa. El presidente ruso goza del más alto respaldo popular a su gestión en su actual tercer mandato y ello es resultado de su actuación en el conflicto con Ucrania y sobre todo de la adhesión exitosa de Crimea a su territorio. Desde el mes de marzo de 2014, la popularidad de Putin ha aumentado $15 \%$ y ha alcanzado $75.7 \%$, el puntaje más alto de los últimos cinco años de acuerdo con el Centro de Investigación de la Opinión Pública de toda Rusia que publicó un informe en su sitio electrónico. ${ }^{22}$ Como ya dijimos, la Unión Europea y el gobierno de Estados Unidos incitaron a las fuerzas de oposición a llevar a cabo estrategias de presiones contra el gobierno de Kiev con el objetivo de derrotarlo y sacar a Ucrania del eje de influencia de Rusia. Sin embargo, hasta el momento se vislumbra un fortalecimiento del liderazgo ruso y un claro margen de maniobra a favor de Moscú en este conflicto que ha trascendido las fronteras del Estado ucraniano.

¿Será que Occidente obtendrá precisamente lo que no aspiraba en relación con Rusia como resultado del conflicto en Ucrania? Es muy probable.

En primer lugar, un evidente fortalecimiento del liderazgo de Rusia tanto en el escenario regional como global. Por sólo citar un

${ }^{20}$ Konstantin Kosachov, "Foreigners View Russia as Unpredictable", Moscow Times, 14 de marzo de 2014, en http://www.themoscowtimes.com/article/49607 6.html

${ }^{21}$ Loc. cit.

22 "El Rating de Putin logra un nuevo récord" (en ruso), Moscú, núm. 2542, 20 de marzo de 2014, en: http:/ / wciom.ru/index.php?id=459\&uid=114752 
ejemplo, la Sexta Cumbre de los BRICs en Fortaleza, Brasil (julio de 2014), resultó un éxito para Putin. En esa ocasión, el presidente ruso recibió un apoyo sólido de los presidentes de los países miembros y se firmó una serie de importantes acuerdos que fortalecen los lineamientos de la política exterior rusa y sus afanes de consolidar un orden mundial multipolar, tanto en el aspecto político, como económico y financiero. Las coincidencias de Putin con Dilma Rousseff y Xi Jinping en los asuntos mundiales son cada vez más sólidas. A diferencia de lo que ha declarado el presidente Obama, Rusia no ha quedado aislada luego del conflicto en Ucrania. Por el contrario, este país gana "simpatías" fuera del marco de la CEI, que ha demostrado ser un mecanismo obsoleto e ineficaz. América Latina se convierte en una región alternativa en mercados para $\mathrm{Ru}$ sia, luego de que países como Brasil, Argentina, Ecuador, Chile, Uruguay y Paraguay hayan demostrado su interés e intención de ofrecer productos, sobre todo alimenticios, que hasta el momento eran importados por Rusia desde los países de la Unión Europea.

En segundo lugar, se inicia una nueva escalada de rearme militar por parte de Rusia en todos los sectores, pero sobre todo el rearme de la aviación estratégica y de la defensa aeroespacial. En una reciente reunión sobre el programa armamentístico ruso para el periodo 2016-2025, Putin planteó la necesidad de conseguir avances importantes en el "desarrollo de todos los componentes de las armas de alta precisión” y subrayó que el país debe producir por sí mismo todos los equipos de importancia vital. ${ }^{23}$ La necesidad de prescindir de las importaciones para la industria de la defensa nacional ya en un corto plazo se convierte en una prioridad para Rusia, luego de las recientes sanciones de Europa y Estados Unidos en este rubro. El Ministerio de Defensa anunció que se prepara una nueva versión de la Doctrina militar de Rusia, la cual será presentada en diciembre próximo. La necesidad de introducir modificaciones a la doctrina militar vigente obedece, según ex-

${ }^{23}$ Vladimir Putin, "Rusia debe tener respuestas adecuadas a todas las amenazas potenciales”, Rossiyskaya Gazieta (Moscú), 10 de septiembre de 2014, en http:/ / es.rbth.com/noticias/2014/09/10/putin_rusia_debe_tener_respuestas_ adecuadas_a_todas_las_amenazas_pot_43493.html 
plicó el subsecretario del Consejo de Seguridad ruso, Mijaíl Popov, al surgimiento de "nuevas amenazas militares", que se manifiestan, por ejemplo, en la crisis ucraniana. ${ }^{24}$

En tercer lugar, como consecuencia del conflicto en Ucrania, Rusia obtiene un mayor y rotundo apoyo interno para su gobierno y, sobre todo, para la figura del presidente Putin, luego de que su tercer mandato fuese resultado de unas elecciones mucho menos favorables que las anteriores. ${ }^{25}$ Putin aspira a reelegirse nuevamente en las elecciones de 2018 por un plazo de otros seis años; este conflicto lo ayudó a ganar apoyo interno, incluso entre sus detractores.

El apoyo de Occidente al nuevo gobierno de Kiev ha logrado que el nacionalismo ruso gane mayor peso e influencia, tanto dentro del marco interno del Estado, como en su proyección exterior. Como señala el profesor de la Universidad de San Francisco, Andrei Tsygankov, "los intentos por explicar la intervención de Putin en Crimea sobre la base de la debilidad económica de Rusia y de su temor a la expansión de la democracia en la región son poco creíbles e ignoran las bases genuinas del nacionalismo ruso y del papel que tiene Occidente en su reciente fortalecimiento". ${ }^{26}$ Existen sectores influyentes alrededor de Putin que presionan desde dentro del Kremlin para lograr que la política exterior y de defensa rusa se vuelva aún más nacionalista y coloque como su máxima prioridad la defensa del interés nacional del Estado ruso, lo cual incluye el rotundo rechazo a la ampliación de la OTAN hacia los territorios fronterizos con Rusia. Precisamente el actual conflicto en Ucrania y las presiones de Occidente contra Rusia han servido de motivación a estos sectores para justificar una línea más conservadora

${ }^{24}$ Alexei Druzhinin, "Russia to Update Military Doctrine by End-2014 - Russian Security Council”, RIA-Novosti, 2 de septiembre de 2014, en http:/ /en.ria.ru/ russia/20140902/192551616/Russia-to-Update-Military-Doctrine-by-End2014-Russian-Security.html

${ }^{25}$ En las últimas elecciones parlamentarias el Partido Rusia Unida disminuyó su representación en la Duma de 315 escaños a 238, por lo que pierde la cómoda mayoría absoluta de que disponía hasta ese momento.

${ }^{26}$ Andrei Tsygankov, "How the West Enabled the Rise of Russian Nationalism", The Nation, EE.UU., 12 de marzo de 2014, en http:/ /www.thenation.com/article/ 178826/how-west-enabled-rise-russian-nationalism\# 
y nacionalista en la actual política exterior. La fuerza más importante dentro de este mecanismo lo constituyen los siloviki, entre los que se encuentran el exministro de Defensa y actual jefe del Consejo Presidencial Serguei Ivanov, el excanciller Igor Ivanov y el presidente de la compañía petrolera estatal Rosneft y exviceprimer ministro, Igor Sechin, quien también había sido considerado el máximo responsable de la política económica durante una década. ${ }^{27}$ La fuerza de los siloviki radica no sólo en que son un grupo de personalidades que mantienen vínculos estrechos con la FSB (órgano sucesor de la кGB), el Ministerio del Interior, el sector militar, sino que también pertenecieron al círculo de San Petersburgo, muy cercano a Putin, que controlan sectores clave de la economía nacional, comparten similares puntos de vista políticos y militares y se encuentran en posiciones estratégicas. ${ }^{28}$ También ocupan posiciones clave en las empresas Rosoboronexport y AlmazAntei, los dos gigantes del sector militar-industrial más importantes del país, en la línea aérea más grande del mundo Aeroflot, en los ferrocarriles rusos y en algunos de los bancos más importantes, como Vneshekonombank, Mezhprombank y Rossiya Bank. ${ }^{29}$

Desde su llegada al poder, Putin señalaba que "aunque Rusia ha dejado de ser un imperio, no ha perdido su condición de gran potencia”. ${ }^{30}$ A partir de ese periodo, la política exterior rusa ha insistido en la definición de dierzhavnost, no como una condición de poderío militar y hegemonía estratégica global, sino como una habilidad de transformarse en un líder mundial con la capacidad de defender sus propios intereses nacionales en el orden internacional desde una postura ofensiva, más que defensiva. Se insiste en

${ }^{27}$ Para conocer más sobre el sector de los siloviki, se recomienda consultar del propio autor, "La evolución de la política exterior de la Federación rusa entre los años 2004 y 2006. ¿Nacionalismo contra pragmatismo?”, Foro Internacional, vol. 47, núm. 188, 2007, pp. 245-268; y El regreso de la eterna Rusia al orden internacional, pp. 270 .

${ }^{28}$ Ian Bremmer y Samuel Charap, "The Siloviki in Putin's Russia: Who They Are and what They Want", Washington Quaterly, vol. 30, núm. 1, invierno de 2007.

${ }^{29}$ Loc. cit.

${ }^{30}$ Vladimir Kozin, "El futuro de la nación con Putin al frente", The Moscow Times, 1 de abril de 2000 . 
la condición de gran potencia de Rusia y en su papel decisivo dentro del orden mundial, en primer lugar, sobre la base de su condición de potencia nuclear.

La sociedad rusa sufrió una profunda decepción moral y material durante la década de 1990, luego del fracaso económico, financiero y social del régimen de Yeltsin. Como consecuencia de ello, el discurso nacionalista de Putin favoreció el fortalecimiento de la desconfianza y rechazo ciudadano a las políticas del gobierno estadounidense en relación con Rusia. La publicación Russia Today publicó recientemente una entrevista al presidente de la Asociación de la Academia de Cuestiones Geopolíticas Konstantin Sivkov, en la que el entrevistado señalaba que era muy probable que el avión malayo hubiese sido derribado por la defensa antiaérea ucraniana y enumeraba una serie de hechos que, en su opinión, así lo demostraban. ${ }^{31}$ Ésta es precisamente la opinión de la mayoría de los ciudadanos rusos en relación con el derribo del avión malayo: culpan al gobierno de Kiev. Por lo tanto, la casi unánime condena a Rusia por este acto terrorista, tanto por parte de los medios de información, como de los círculos políticos occidentales logró fortalecer la cohesión interna de la sociedad rusa y el apoyo a su gobierno. Esto favoreció a Putin.

Desde un inicio, se vislumbraban ciertos riesgos que no fueron tomados en cuenta por las potencias europeas y por el gobierno de Obama, pero que vinieron a complicar aún más el escenario de negociación, tanto interno en Ucrania, como con el gobierno de Rusia. En primer lugar, el hecho de que precisamente las fuerzas que lideraban las manifestaciones en Kiev estuviesen mayormente compuestas por organizaciones y partidos de tendencia de centro-derecha, pero que también participaban fuerzas radicales de extrema derecha -con algunos representantes incluso catalogados como de tendencia neofascista (Partido Sbovoda)-, despertó la desconfianza en parte de la sociedad ucraniana y sobre todo del gobierno ruso, que las calificó de neofascistas y extremistas. Lo que

31 "Ciertos hechos indican que el avión malayo fue derribado por el ejército ucraniano”, en Russia Today (Moscú), 18 de julio de 2014, en: http://actualidad. rt.com/actualidad/view/134346-hechos-indicar-avion-derribar-ucraniana 
se conoció como el Praviy Sektor dentro de la oposición ucraniana era una especie de confederación de organizaciones y agrupaciones de tendencia de derecha y extrema derecha, tales como los Patriotas de Ucrania, la Asamblea Social-Nacional, la Asociación de todos los Ucranianos de Stepan Bandera "Trident", 32 la organización de Kiev "White Hummer", entre otras. La premisa de Bandera era justamente que

si los no ucranianos comprendían la urgencia de los ucranianos en consolidar su Estado nación y estaban dispuestos a ayudarnos en su lucha, entonces nosotros estaríamos dispuestos a colaborar con ellos también; si eran neutrales y no entorpecían nuestra lucha nacionalista, entonces nosotros seríamos neutrales también con ellos; pero si rechazaban nuestro derecho a crear un Estado nación independiente y trabajaban contra nosotros, entonces seríamos hostiles con ellos. ${ }^{33}$

Durante la Guerra Fría, el gobierno de Estados Unidos utilizó a los veteranos de las fuerzas de Bandera en su lucha contra la Unión Soviética, sin tomar en cuenta su colaboración con los nazis. En la actualidad, las organizaciones del Praviy Sektory de Svoboda utilizan y difunden muchas de las convicciones, prácticas y símbolos neonazis. El uso de la simbología, bandera y saludos nazis tales como "Gloria a Ucrania-Gloria a los Héroes" recuerda a la etapa del movimiento de Bandera, quien juró fidelidad a Hitler y luchó contra los partisanos soviéticos. ${ }^{34}$ Estos dos movimientos extremistas fortalecidos luego del golpe de Estado en Ucrania se des-

${ }^{32}$ Stepan Bandera, líder del nacionalismo ucraniano, quien colaboró con los nazis y se enfrentó al ejército soviético durante la década de los 40 y 50 , fue convertido en héroe nacional luego de ser asesinado por un agente de la KGB en Múnich en 1959.

33 Oleg Shynkarenko, "Can Ukraine Control Its Far Rigth Ultranationalist?", The Daily Beast, 1 de marzo de 2014, en http:/ / www.thedailybeast.com/articles /2014 /03/01/can-ukraine-control-its-far-right-ultranationalists.html

34 Serguei Markov, “After Kiev Coup, the West Will Focus on Moscow”, The Moscow Times, 27 de marzo de 2014, en http://www.themoscowtimes.com/opinion /article/after-kiev-coup-the-west-will-focus-on-moscow/496915.html 
tacan por sus tendencias antisemitas, el odio a los pueblos vecinos postsoviéticos, la propagación de los sentimientos de rusofobia, la lucha violenta en contra de los opositores a sus ideas, y la glorificación de los veteranos nazis, así como la negación de los crímenes cometidos por ellos. ${ }^{35}$

Algo preocupante era que la extrema derecha había asegurado un predominio y un nivel de representación importante en los órganos representativos de poder que no tenía paralelo alguno con el resto de los países europeos, donde sí existen partidos de extrema derecha representados en el parlamento, pero no en el gobierno como fue el caso de este país. Por ejemplo, los puestos de Ministro de Defensa y de Presidente del Consejo de Defensa y de Seguridad Nacional del gobierno de transición fueron ocupados por miembros del partido Sbovoda, a la vez que otros puestos importantes del gobierno eran ocupados también por miembros de este partido de extrema derecha, así como también en las altas instancias de los gobiernos regionales de las provincias del oeste. Dentro del parlamento, uno de los representantes de este partido, Mijaíl Golovko, señalaba que "nosotros recuperaremos nuestra condición de potencia nuclear y esto cambiará las condiciones de negociación. Ucrania dispone de todos los medios tecnológicos necesarios para crear un arsenal nuclear propio en un plazo de 3 a 6 meses". ${ }^{36}$ Esta retórica belicista que contradice el compromiso adoptado por Ucrania en 1994 con la firma del acuerdo internacional de no proliferación nuclear no es nuevo para los dirigentes de Svoboda. Su máximo líder, Oleg Tyagnibok ya había prometido regresar al país a su condición de potencia nuclear, durante su campaña en las elecciones presidenciales del año 2010. Un resultado favorable para intentar un clima de cierta moderación interna ha sido las recientes elecciones parlamentarias (26 de octubre). En esta ocasión, tanto los partidos de extrema derecha como el Partido Comunista quedaron fuera del parlamento al no lograr el mínimo de $5 \%$

${ }^{35}$ Loc. cit.

${ }^{36}$ Gleb Goronin, "Calls in Kiev to 'Regain Nuclear Status in Six Months”, Russia Today (Moscú), 1 de marzo de 2014, en http:/ /rt.com/news/ukraine-nuclear-arsenal-threat-314/ 
de los votos, lo cual indica que la voluntad de los ciudadanos ucranianos es apoyar un régimen mediador, tanto en el plano interno como en sus relaciones exteriores. Sin embargo, en la provincia de Donetsk se votó solamente en 12 de los 21 distritos electorales y en Lugansk en 5 de 11, por lo que el porcentaje de abstención en las elecciones generales fue el más alto de los últimos años. De igual forma, apenas una semana después se celebraron elecciones independientes en esas regiones del Este, las cuales fueron apoyadas por el Kremlin y no han sido reconocidas por el gobierno de Kiev. ${ }^{37}$

\section{LAS POSTURAS de PREsión de Estados Unidos HaCia Moscú y SUS RESUltados AMBIVALENTES}

En otro orden de cosas, un error geopolítico del presidente Obama ha sido el liderar y avanzar en la aplicación de sanciones económicas y financieras contra Rusia, las cuales no tuvieron las repercusiones esperadas en el plazo inmediato. Los mercados de Asia, Medio Oriente y América Latina ofrecen alternativas favorables para el comercio con Rusia y mantienen su interés en profundizar los lazos comerciales bilaterales. Rusia es un país que exporta fundamentalmente recursos naturales (gas, petróleo, minerales estratégicos) que no pueden ser reemplazados fácilmente, lo que la hace menos vulnerable. Por el contrario, Europa importa cerca del $40 \%$ del gas de Rusia y varios de los países de Europa del Este, cerca del 100\%, lo cual les dificulta encontrar un mercado alternativo a Rusia, ni siquiera en un mediano plazo. Alemania, Francia e Italia son los países que importan mayor cantidad de gas natural desde Rusia y serían los más afectados el próximo invierno en caso de llevarse a cabo un bloqueo energético desde Moscú. Una guerra comercial entre Rusia y la Unión Europea sería muy complicada para la primera, pero también para la Unión Europea, que apenas va saliendo de la crisis económica, financiera y fiscal de 2008. Existe un estudio

${ }^{37}$ En estas elecciones participó $52.45 \%$ del electorado, cifra inferior a las anteriores elecciones parlamentarias (2012) y a las presidenciales de mayo (2014). Pilar Bonet, "Resultados de las elecciones en Ucrania", El País, 27 de octubre de 2014. 
reciente de la Comisión Europea que pronostica un descenso anual del PIB de la Unión Europea entre un 0.5 y $1 \%$ mientras dure el enfrentamiento comercial y económico con Rusia. ${ }^{38}$

La promesa hecha por el presidente Obama a la Unión Europea de suministrarles gas -basada en su interés de penetrar también las reservas ucranianas- se vislumbra remota e ilusoria, pues el gas licuado estadounidense difícilmente podría convertirse en el sustituto del ruso dentro del mercado europeo ni siquiera en un mediano plazo, tomando en cuenta los altos costos de transportación y comercialización. Asimismo, aunque el comercio de Rusia con Estados Unidos es mínimo, este país es el cliente más importante de la Unión Europea en cuestiones comerciales y de inversiones, por lo cual de mantenerse la política de sanciones contra Rusia estos países también se verían afectados. Los casos de Vietnam, Tailandia, Indonesia y Myanmar constituyen claros ejemplos de oportunidades potenciales para Rusia en el orden comercial en Asia. Precisamente China y Japón han despuntado como los posibles sustitutos de una parte del comercio de energéticos que actualmente mantiene Rusia con países de la Unión Europea, en caso de que las relaciones políticas y comerciales con Europa continuasen empeorando.

El caso de China constituye el más representativo. A partir del primer semestre de 2014, las relaciones comerciales bilaterales se han profundizado de manera significativa. Se firmaron varios acuerdos estratégicos no solamente en la esfera de los energéticos, sino también en la industria militar, de electricidad y de construcción de maquinarias. Durante la visita de Putin a China (mayo de 2014), se firmó un Comunicado conjunto para profundizar la cooperación económica y comercial en el sector energético y aumentar los suministros de gas, petróleo, electricidad y carbón rusos a China. En el marco de las reuniones gubernamentales entre delegaciones comerciales de ambos países, la compañía rusa Novatek y la china CNPC firmaron un contrato para la entrega de tres millones de toneladas anuales de gas natural licuado ruso. ${ }^{39}$ Gazprom y la compañía china

38 Lucía Abellán, "La UE propone por primera vez sanciones económicas a Rusia”, en El País, 22 de julio de 2014.

${ }^{39}$ Jacob Heilbrunn, “Putin’s Audacious \$400 Billion Gas Deal”, The National 
CNPC suscribieron el contrato mencionado para el suministro a China de 38000 millones de metros cúbicos de gas anuales durante un periodo de treinta años, por un valor total de 400000 millones de dólares. En 2014 se firmaron más de 40 documentos conjuntos en las esferas de transporte, energía, infraestructuras, industria automovilística y construcción aeronáutica. China es el principal socio comercial de Rusia; la intención de ambos países es estrechar aún más los nexos bilaterales. La circulación de mercancías bilateral alcanza la cifra de 90000 millones de dólares anuales y se ampliará en los próximos años. ${ }^{40}$

\section{Conclusiones}

El descontento de la sociedad ucraniana estaba directamente relacionado con la pésima gestión económica y social de los gobiernos ucranianos previos, así como por la corrupción del sistema político imperante, incluido en primer lugar el del régimen de Yanukovich, pero también el de su antecesor Yuschenko. Los indicadores socioeconómicos de las últimas dos décadas corroboran esta afirmación. En el año 1989, el PIB global de Ucrania rebasaba ampliamente el de su vecina Polonia. En la actualidad, el PIB global de Polonia -país $20 \%$ más pequeño que Ucrania- es tres veces superior al de Ucrania. Polonia se sitúa en el cuartil superior (más favorable) en relación con los indicadores de percepción de corrupción de Transparencia Internacional, mientras que Ucrania ocupa el lugar 144 de 177, al nivel de Nigeria e Irán. ${ }^{41}$

A pesar del clima tenso y ríspido que se mantiene en Ucrania, no se vislumbra un enfrentamiento militar directo entre Rusia y Occidente, pero tampoco lo hubo durante la Guerra Fría. Sin em-

Interest, 21 de mayo de 2014, en http:/ / nationalinterest.org/blog/jacob-heilbrunn /putins-audacious-400-billion-gas-deal-10505

40 Alexei Druzhinin, "Gazprom firma el contrato de gas con China", Agencia de noticias RIA-Novosti, Shanghái, 21 de mayo de 2014, en http://sp.ria.ru/economy/20140521/160155188.html

${ }^{41}$ Richard Burt y Lee Feinstein, "Ukraine's Future”, The National Interest, Washington, 28 de febrero de 2014. 
bargo, tampoco se vislumbra en un corto plazo un clima de negociación efectivo que propicie un escenario de paz y entendimiento mutuo duradero y estable. Por el contrario, de mantenerse el actual clima de tensión y confrontación en Ucrania se lograría una influencia negativa en el orden regional europeo y en el entorno mundial, sobre todo en los asuntos del comercio y la economía, así como un reacomodo en las alianzas estratégicas regionales y globales. La reciente gira del presidente ruso por América Latina y su liderazgo en la Cumbre de los países BRICs corroboran este escenario. Las actuales posturas de contención y aislamiento en relación con Rusia llevadas a cabo por Estados Unidos y Europa despiertan también la cautela y desconfianza de otras potencias regionales más cercanas a Rusia como son China, Brasil e incluso Irán.

La profundización del conflicto en Ucrania ha eliminado el frágil clima de entendimiento y compromiso que se había logrado alcanzar entre Rusia y Occidente, luego de finalizada la Guerra Fría. A pesar de que este clima de diálogo y negociación bilateral resultó inestable, intermitente y frágil, logró evitar el resurgimiento de un clima de enemistad y desconfianza similar al que existió durante la Guerra Fría. Si bien la integración entre Rusia y Occidente nunca llegó a consolidarse, sí se había logrado establecer una atmósfera de relativa interacción y colaboración en el escenario internacional e incluso de trabajo coordinado en frentes comunes como es el de la lucha antiterrorista o el desarme nuclear. Por el contrario, la actual crisis ucraniana despierta nuevamente la desconfianza, la rivalidad, la competencia geopolítica e incluso la confrontación diplomática y comercial entre ambas partes. Europa nuevamente se coloca en una postura de rehén de Estados Unidos en relación con Rusia, lo cual nos recuerda el año 2008 cuando el gobierno de George W. Bush anunció el emplazamiento de bases de radares y de un escudo de sistema antimisiles en países europeos (inicialmente Polonia y República Checa y después en Rumania y Bulgaria) que fue considerado por Rusia como una amenaza directa a su seguridad nacional e integridad territorial. Este factor unido a la creación ese mismo año del Estado independiente de Kosovo y al decisivo apoyo brindado por parte de Estados Unidos, la otan y la Unión Europea en este proceso, provocó 
un enfriamiento de las relaciones entre Rusia y Occidente, pero sin alcanzar los niveles actuales de confrontación.

El presidente Putin ha reiterado en varias ocasiones su idea sobre la importancia de consolidar la Gran Europa. En un artículo publicado en mayo de 2005, afirmaba:

estoy profundamente convencido que la Gran Europa Unida desde el Atlántico hasta los Urales, y de hecho incluyendo el territorio ruso hasta el Océano Pacífico, ofrece una oportunidad única a todas las naciones del continente, incluida la nación rusa. [...] Creemos en que los esfuerzos de Rusia para desarrollar lazos de integración tanto con los Estados miembros de la Unión Europea como con los Estados miembros de la CEI constituyen un proceso único y orgánico que debe conducir a una expansión considerable de los espacios comunes armoniosos de seguridad, democracia y cooperación en los negocios en toda nuestra gran región. ${ }^{42}$

Las ideas principales de este artículo eran dos: primero que el liderazgo de Rusia en los países de la cei no entraba en contradicción con la integración de todo el continente europeo, incluida Rusia y, segundo, que se excluía a Estados Unidos en este proceso de construcción de un espacio único intraeuropeo. ${ }^{43}$ Según Putin, en Europa pueden coexistir dos centros de poder claramente definidos: la Europa comunitaria bajo la égida de Alemania y la Europa de la región euroasiática bajo el liderazgo de Rusia. Para Moscú, Ucrania siempre ha estado incluida en el segundo grupo.

La crisis en Ucrania se ha ideologizado tanto dentro como fuera del país, lo cual nos recuerda el clima tenso de desconfianza y enfrentamiento ideológico que existía durante la Guerra Fría. ¿Por qué Occidente debe tomar decisiones y dictar soluciones en un

42 V. Putin, "Las lecciones de la victoria sobre el Nazismo- hacia la construcción conjunta de un futuro de seguridad para la humanidad" (en ruso), 7 de mayo de 2005, Ministerio de Asuntos Exteriores de Rusia, en http://www.mid.ru/nspobeda.nsf/304a70a9f8af4383c3256eda00378036/c3256eda00375761c3256ffb00 30159b?OpenDocument

${ }^{43}$ Marek Menkiszak, “Greater Europe Putin's vision of Europe AN (dis)integration”, Center for Eastern Studies, Varsovia, núm. 46, octubre de 2013, p. 12. 
conflicto que no les compete ni afecta de manera directa? Esto dificulta el logro de un clima de entendimiento y diálogo entre las partes que conlleve a una solución negociada. Ucrania no debería quedar dividida en dos Estados independientes; ésa quizás sería una solución extremista y forzada, que no conduciría a una paz estable y duradera. Por el contrario, se aseguraría el terreno propicio para un conflicto permanente entre las partes involucradas. Tan difícil y complicado sería buscar un total distanciamiento político, económico, administrativo y cultural entre Ucrania y Rusia, como fomentar una separación entre los territorios del este y del oeste del actual Estado ucraniano. Ninguno de estos procesos lograría consolidar una paz duradera en la región. ¿Buscar una mayor federalización del Estado en el marco de las leyes ya existentes, pero sin llegar a establecer una separación completa de los territorios del este? Quizás ésta sería la solución más conveniente para todas las partes involucradas; pero sobre todo para los propios ucranianos, que en definitiva siguen siendo la parte más importante del conflicto. Ese amable y pacífico pueblo que hoy sufre una guerra inmerecida.

\section{BibLIOGRAFÍA}

a) Libros

Mearsheimer, John J., La tragedia de la politica de las grandes potencias (The Tragedy of Great Power Politics), Nueva York, W. W. Norton, 2001.

Sánchez Ramírez, Pablo Telman, Razón y poder: Rusia, una potencia en el siglo XxI, México, Miguel Ángel Porrúa / Tecnológico de Monterrey, 2005. , El regreso de la eterna Rusia al orden internacional. ¿Confrontación o negociación con Occidente?, México, Tecnológico de Monterrey / Montiel \& Soriano Editores, 2010.

b) Artículos en revistas y periódicos

Abellán, Lucía, "La UE propone sanciones a Rusia que superan los 10000 millones", El País, 24 de julio de 2014. 
— sia”, El País, 22 de julio de 2014.

Bremmer, Ian y Samuel Charap, "The siloviki in Putin's Russia: Who They Are and what They Want", Washington Quaterly, vol. 30, núm. 1, invierno de 2007.

Burt Richard y Lee Feinstein, "Ukraine's Future", The National Interest, Washington, 28 de febrero de 2014.

Kozin, Vladimir, "El futuro de la nación con Putin al frente", The Moscow Times, 1 de abril de 2000.

Mearsheimer, John J., "Getting Ukraine Wrong", The International New York Times, 14 de marzo de 2014.

Menkiszak, Marek, "Greater Europe Putin's vision of Europe AN (dis)integration”, Center for Eastern Studies, Varsovia, núm. 46, octubre de 2013, p. 12.

Sánchez Ramírez, Pablo Telman, "La evolución de la política exterior de la Federación rusa entre los años 2004 y 2006. ¿Nacionalismo contra pragmatismo?", Foro Internacional, vol. 47, núm. 188, 2007, pp. 245268.

\section{c) Páginas electrónicas}

Abellán, Lucía, "Ucrania y la UE firman el pacto de libre comercio que desató la crisis con Rusia”, El País, 27 de junio de 2014, en http:// internacional.elpais.com/internacional/2014/06/27/actualidad/ 1403853122_102740.html [2 de julio de 2014].

Druzhinin, Alexei, "Gazprom busca cotizar en la bolsa de Hong Kong", Agencia de noticias RIA-Novosti, Moscú, 26 de junio de 2014, en http://sp.ria.ru/economy/20140626/160562654.html

_ , "Gazprom firma el contrato de gas con China", Agencia de noticias RIA-Novosti, Shanghái, 21 de mayo de 2014, en http://sp.ria.ru/ economy/20140521/160155188.html

-, "Russia to Update Military Doctrine by End-2014 - Russian Security Council", RIA-Novosti, 2 de septiembre de 2014, en http:// en.ria.ru/russia/20140902/192551616/Russia-to-Update-MilitaryDoctrine-by-End-2014-Russian-Security.html

Goronin, Gleb, "Calls in Kiev to 'Regain Nuclear Status in Six Months'”, 
Russia Today (Moscú), 1 de marzo de 2014, en http://rt.com/news/ ukraine-nuclear-arsenal-threat-314/

Heilbrunn, Jacob, “Putin’s Audacious \$400 Billion Gas Deal”, The National Interest, Washington, 21 de mayo de 2014, en http:/ / nationalinterest. org/blog/jacob-heilbrunn/putins-audacious-400-billion-gasdeal-10505

Kosachov, Kosachov, "Foreigners View Russia as Unpredictable", Moscow Times, 14 de marzo de 2014, en http://www.themoscowtimes.com/ article/496076.html

Lutz, Christian, "Cómo Rusia responderá a las nuevas sanciones de la Unión Europea”, Agencia de noticias RIA-Novosti, Moscú, 12 de septiembre de 2014, en http: / / sp.ria.ru/revista_de_prensa/20140912/16 1793263.html

Markov, Serguei, “After Kiev Coup, the West Will Focus on Moscow”, The Moscow Times, 27 de marzo de 2014, en http:/ / www.themoscowtimes. com/opinion/article/after-kiev-coup-the-west-will-focus-on-moscow/496915.html

Meinung, Hubert, "Russland wendet sich wieder altem Denken zu" ("Rusia regresa al antiguo pensamiento"), entrevista realizada para F.A.Z. interview, Alemania, 16 de mayo de 2014 en http:/ / www.faz.net/aktuell/ politik/kanzlerin-merkel-im-f-a-z-gespraech-russland-wendet-sichwieder-altem-denken-zu-12941544.html

Osborn, Andrew, “Crimea, a Symbol of Russian Identity Vital for Putin's Legacy”, 13 de marzo de 2014, cable de Agencia de Noticias Reuters, en http://in.reuters.com/article/2014/03/13/ukraine-crisis-crimea -putin-idINDEEA2C08H20140313

Putin, Vladimir, "Las lecciones de la victoria sobre el Nazismo- hacia la construcción conjunta de un futuro de seguridad para la humanidad" (en ruso), 7 de mayo de 2005, Ministerio de Asuntos Exteriores de Rusia, en http:/ / www.mid.ru/ns-pobeda.nsf/304a70a9f8af43 83c3256eda00378036/c3256eda00375761c3256ffb0030159b?Open Document

, "Rusia debe tener respuestas adecuadas a todas las amenazas potenciales", Rossiyskaya Gazieta (Moscú), 10 de septiembre de 2014, en http://es.rbth.com/noticias/2014/09/10/putin_rusia_debe_tener_respuestas_adecuadas_a_todas_las_amenazas_pot_43493.html [15 de septiembre de 2014]. 
Rozin, Igor, "Putin solicita a los senadores la aprobación para el uso de la fuerza militar en Ucrania”, Rossiyskaya Gazieta (Moscú), 1 de marzo de 2014, en http://es.rbth.com/noticias/2014/03/01/putin_pide_al_ senado_ruso_autorizacion_para_emplear_tropas_rusas_en_37783. html

Schwarz, Karl-Peter, "Krise in der Ukraine. Im Kreml lacht man über Sanktionen" ("El Kremlin se ríe de las sanciones"), Pressburg, Alemania, 15 de mayo de 2014, en http:/ /www.faz.net/aktuell/politik/ europaeische-union/krise-in-der-ukraine-im-kreml-lacht-man-uebersanktionen-12940541.html

Simes, Dimitri, "How Obama Is Driving Russia and China Together", The National Interest, Washington, 24 de junio de 2014, en http:/ / nationalinterest.org/feature/how-obama-driving-russia-china-together10735

Shynkarenko, Oleg, "Can Ukraine Control Its Far Rigth Ultranationalist?”, The Daily Beast, 1 de marzo de 2014, en http://www.thedailybeast. com/articles/2014/03/01/can-ukraine-control-its-far-right-ultranationalists.html [10 de abril de 2014].

Tapia, Antonio, "Obama busca contener a Rusia y anuncia más tropas para Europa", La Tercera, Chile, 4 de junio de 2014, en http:/ / diario. latercera.com/2014/06/04/01/contenido/mundo/8-166017-9-obama-busca-contener-a-rusia-y-anuncia-mas-tropas-para-europa.shtml

Tsygankov, Andrei, "How the West Enabled the Rise of Russian Nationalism", The Nation, 12 de marzo de 2014, en http://www.thenation. com/article/178826/how-west-enabled-rise-russian-nationalism\#

Volokin, Yevgueni, "Ucrania: 46 personas mueren en Odesa en los enfrentamientos y un incendio intencional", Russia Today (Moscú), 2 de mayo de 2014, en http:/ /actualidad.rt.com/actualidad/view/127017odesa-incendio-casa-sindicatos-disturbios

\section{d) Documentos}

Concepto de la Política Exterior de la Federación Rusa (traducción no oficial del ruso), Boletín informativo del Ministerio de Asuntos Exteriores de la Federación Rusa, Departamento de Información y Prensa, Moscú, 18 de febrero de 2013, p. 6. 